\title{
Online Facility Assignment
}

\author{
Abu Reyan Ahmed ${ }^{1}$, Md. Saidur Rahman ${ }^{2}$ and Stephen Kobourov ${ }^{1}$ \\ ${ }^{1}$ Dept. of Computer Science, University of Arizona, \\ \{abureyanahmed@email,kobourov@cs\}.arizona.edu \\ ${ }^{2}$ Dept. of Computer Science and Engineering, Bangladesh University of Engineering \\ and Technology, saidurrahman@cse.buet.ac.bd
}

\begin{abstract}
We consider the online facility assignment problem, with a set of facilities $F$ of equal capacity $l$ in metric space and customers arriving one by one in an online manner. We must assign customer $c_{i}$ to facility $f_{j}$ before the next customer $c_{i+1}$ arrives. The cost of this assignment is the distance between $c_{i}$ and $f_{j}$. The total number of customers is at most $|F| l$ and each customer must be assigned to a facility. The objective is to minimize the sum of all assignment costs. We first consider the case where facilities are placed on a line so that the distance between adjacent facilities is the same and customers appear anywhere on the line. We describe a greedy algorithm with competitive ratio $4|F|$ and another one with competitive ratio $|F|$. Finally, we consider a variant in which the facilities are placed on the vertices of a graph and two algorithms in that setting.
\end{abstract}

\section{Introduction}

Let $F=\left\{f_{1}, f_{2}, \cdots, f_{|F|}\right\}$ be a set of facilities, each with capacity $l$. We first consider the case when facilities are placed on line $L$, such that the distance between every pair of adjacent facilities is $d$, where $d$ is a constant. An input sequence $I=\left\{c_{1}, c_{2}, \cdots, c_{n}\right\}$ is a set of $n$ customers who arrive one at a time in an online manner, with $c_{i}$ corresponding to the location of customer $i$ on the line $L$. The distance between a customer $c_{i}$ and a facility $f_{j}$ is the Euclidean distance between $c_{i}$ and $f_{j}$. We later consider the case in which the facilities are located on the vertices of a graph $G=(V, E)$ and customers appear on the vertices of $G$. In that case, the distance between a customer $c_{i}$ and a facility $f_{j}$ is equal to the number of edges in the shortest path between $c_{i}$ and $f_{j}$.

Any algorithm for this problem must assign a customer $c_{i}$ to a facility $f_{j}$ before the next customer $c_{i+1}$ arrives, where the cost of that assignment is the distance between $c_{i}$ and $f_{j}$. The total number of customers is at most $|F| l$ as every facility can serve at most $l$ customers and each customer must be assigned to a facility. The objective is to minimize the total cost of all assignments. We call this problem the online facility assignment problem. This problem arises naturally in different practical applications, such handling online orders for a restaurant with multiple locations, and handling network packets in network with multiple routers.

${ }^{1}$ Work on this project was funded in part by NSF grant CCF-AF 1712119. 


\section{$1.1 \quad$ Related Work}

In the classical facility location problem, customer locations are known ahead of time and the objective is to compute locations for a set of facilities that can handle all the customers. The Fermat-Weber problem is considered the first facility location problem, studied as early as in the 17th century; see the survey of Drezner [9] and the textbook by Drezner and Hamacher [10]. A recently proposed facility location variant is the $r$-gathering problem. An $r$-gathering of a set of customers $C$ for a set of facilities $F$ is an assignment of $C$ to open facilities $F^{\prime} \subset F$ such that $r$ or more customers are assigned to each open facility. Armon [3] describes a simple 3-approximation algorithm for this problem. Akagi and Nakano [1] provide an $O((|C|+|F|) \log |C|+|F|)$ time algorithm to solve the $r$-gathering problem when all customers in $C$ and facilities in $F$ are on the real line.

The online facility assignment problem is also related to the $k$-server problem proposed by Manasse et al. [16], which requires scheduling the movement of a set of $k$ servers, represented as points in a metric space, in order to handle requests that are also in the form of points in the space. For each request, the algorithm must determine which server to move to the requested point, with the goal of minimizing the total distance covered by all servers. This problem has been extensively studied [14, 18, 7, 8, 5, 6, 15].

Despite similarities, the $k$-server problem and the online facility assignment problem are different. The servers in the $k$-server problem are movable, whereas the positions of facilities are fixed in the online facility assignment problem. Therefore, a customer placed very close to a previous customer is easily served in the server problem which is not true for the facility assignment problem.

The facility assignment problem is also related to the matching problem [17], which is one of the fundamental and well-studied optimization problems. The facility assignment problem can be seen as a generalization of the matching problem, where each facility has capacity $l \geq 1$. Online variants of matching have been extensively studied $[11,13,12,4,2]$. Kao et al. [12] provide a randomized lower bound of 4.5911 for online matching on a line. We provide a randomized algorithm, which is $\frac{9}{2}$-competitive for a class of input sequences. Antoniadis et al. [2] describe an $o(n)$-competitive deterministic algorithm for online matching on a line.

\subsection{Our Contributions}

We first consider the case where both the facilities $F$ and the customers $C$ are on a line. We propose Algorithm Greedy and show that it has competitive ratio $4|F|$. Introducing randomization in Algorithm Greedy leads to an improved performance of $9 / 2$ for a special class of input instances. We then describe Algorithm OptimalFill and show it has competitive ratio $|F|$.

We next consider the case where both the facilities $F$ and the customers $C$ are located on the vertices of an unweighted graph $G=(V, E)$. We show that Algorithm Greedy has competitive ratio $2|E(G)|$ and Algorithm Optimal-Fill has competitive ratio $|E(G)||F| / r$, where $r$ is the radius of $G$. Finally, we consider the case where a customer leaves after receiving service at a facility. We define service time as the amount of service time needed, and study the facility assignment problem with limited service time. 
The rest of this paper is organized as follows. In Section 2 we provide basic definitions. In Section 3 we study the online facility assignment problem on a line. In Section 4 we study the graph version of the problem. In Section 5 we introduce a service time parameter $t$ in our model and show that no deterministic algorithm is competitive when $t=2$.

\section{Preliminaries}

A graph $G=(V, E)$ consists of a finite set $V$ of vertices and a finite set $E$ of edges; each edge is an unordered pair of vertices. We often denote the set of vertices $G$ by $V(G)$ and the set of edges by $E(G)$. We say $G$ is unweighted if every edge of $G$ has equal weight. Let $u$ and $v$ be two vertices of $G$. If $G$ has a $u, v$-path, then the distance from $u$ to $v$ is the length of a shortest $u, v$-path, denoted by $d_{G}(u, v)$ or simply by $d(u, v)$. If $G$ has no $u, v$-path then $d(u, v)=\infty$. The eccentricity of a vertex $u$ in $G$ is $\max _{v \in V(G)} d(u, v)$ and denoted by $\epsilon(u)$. The radius $r$ of $G$ is $\min _{u \in V(G)} \epsilon(u)$ and the diameter of $G$ is $\max _{u \in V(G)} \epsilon(u)$. The center of $G$ is the subgraph of $G$ induced by vertices of minimum eccentricity.

In the online facility assignment problem, we are given a set of facilities $F=$ $\left\{f_{1}, f_{2}, \cdots, f_{|F|}\right\}$ of equal capacity $l$ in a metric space, and an input sequence of customers $I=\left\{c_{1}, c_{2}, \cdots, c_{n}\right\}$ which is a set of $n$ customers who arrive one at a time in an online manner, with $c_{i}$ corresponding to the location of customer $i$ in the given space. We say an input $I$ is well distributed if there is at least one customer between any two adjacent facilities. The capacity of a facility is reduced by one when a customer is assigned to it. We denote the current capacity of facility $f_{i}$ by capacity $_{i}$. A facility $f_{i}$ is called free if capacity $_{i}>0$. Any algorithm ALG for this problem must assign a customer $c_{i}$ to a free facility $f_{j}$ before a new customer $c_{i+1}$ arrives. The cost of this assignment is the distance between $c_{i}$ and $f_{j}$, which is denoted by distance $\left(f_{j}, c_{i}\right)$. The total number of customers is, at most, $|F| l$ and each customer must be assigned to a facility. For any input sequence of customers $I$, Cost_ALG $(I)$ is defined as the total cost of all assignments made by ALG. The objective is to minimize Cost_ALG $(I)$.

We say an algorithm is optimal if, for any input sequence of customers, the total cost of the assignment it provides is the minimum possible. We denote an optimal algorithm by OPT. An online algorithm ALG is $c$-competitive if there is a constant $\alpha$ such that, for all finite input sequences $I$,

$$
\text { Cost_ALG }(I) \leq c \text {.Cost_OPT }(I)+\alpha \text {. }
$$

The factor $c$ is called the competitive ratio of ALG. When the additive constant $\alpha$ is less than or equal to zero (i.e., Cost_ALG $(I) \leq c$.Cost_OPT $(I)$ ), we may say, for emphasis, that ALG is strictly c-competitive. An algorithm is called competitive if it attains a constant competitive ratio $c$. Although $c$ may be a function of the problem parameters, it must be independent of the input $I$. The infimum over the set of all values $c$ such that ALG is c-competitive is called the competitive ratio of ALG and is denoted by $\mathcal{R}$ (ALG). 


\section{Facility Assignment on a Line}

Let $F=\left\{f_{1}, f_{2}, \cdots, f_{|F|}\right\}$ be a set of facilities placed on a line, such that the distance between every pair of adjacent facilities is $d$, where $d$ is a constant. An input sequence $I=\left\{c_{1}, c_{2}, \cdots, c_{n}\right\}$ is a set of $n$ customers who arrive one at a time in an online manner, with $c_{i}$ corresponding to the location of customer $i$ on the line. In Section 3.1 we describe Algorithm Greedy with competitive ratio $4|F|$. In Section 3.2 we introduce randomization to Algorithm Greedy and show that it is $\frac{9}{2}$-competitive for a special class of input sequences. In Section 3.3 we describe Algorithm Optimal-Fill and show it has competitive ratio $|F|$.

\subsection{Algorithm Greedy}

Here we describe and analyze the natural greedy algorithm, which assigns each customer to the nearest free facility.

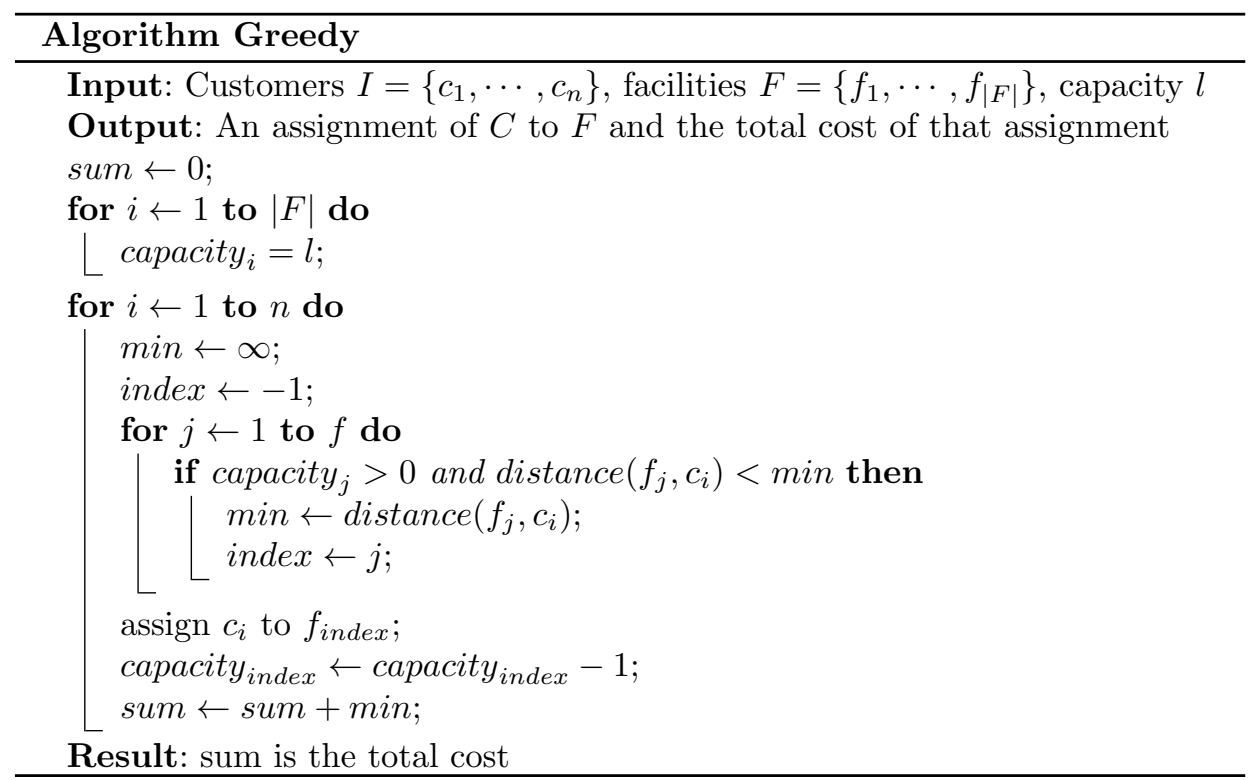

We can analyze the online algorithm in the context of a game between an online player and a malicious adversary. The online player runs the online algorithm on an input created by the adversary. The adversary, based on the knowledge of the online algorithm, constructs the worst possible input (i.e., one that maximizes the competitive ratio). Consider Algorithm Greedy above and the adversary strategy of making an instance very costly for Algorithm Greedy but, at the same time, inexpensive for OPT. The following lemma gives a lower bound for OPT's cost.

Lemma 1. Let $d$ be the distance between all adjacent facilities. If the assignments of OPT and Algorithm Greedy are not the same, then OPT's cost is at least $\frac{d}{2}$.

Proof. Let $c_{x}$ be the first customer for which the assignments of OPT and Algorithm Greedy differ. The optimal cost for assigning $c_{x}$ is at least $\frac{d}{2}$. Hence the total optimal cost is at least $\frac{d}{2}$. 
The following theorem determines the worst input sequence an adversary can construct for Algorithm Greedy and provides a competitive ratio.

Theorem 1. Let $F=\left\{f_{1}, f_{2}, \cdots, f_{|F|}\right\}$ be a set of facilities placed on the line, such that the distance between every pair of adjacent facilities is $d$, where $d$ is a constant. Then $\mathcal{R}$ (Algorithm Greedy) $\leq 4|F|$.

Proof. Recall the definition of a well distributed input sequence, namely that there is at least one customer between any two adjacent facilities. When the metric space is a line, all customers have cost less than $d$ in the optimum assignment of a well distributed input sequence. However, if the input sequence is not well distributed, there are some customers with assignment cost greater than $d$. We consider these two cases separately. For both cases, assume now that the facilities have unit capacity. Later we will also deal with the case for capacity $l$, where $l>1$.

Let $f_{l}$ is the leftmost facility and $f_{r}$ be the rightmost facility. Consider a customer $c$ who appears to the left of $f_{l}$. The distance between $c$ and $f_{l}$ is distance $\left(f_{l}, c\right)$. Both Cost_Algorithm_Greedy $(I)$ and Cost_OPT $(I)$ must pay the amount distance $\left(f_{l}, c\right)$. The ratio of Cost_Algorithm_Greedy $(I)$ to Cost_OPT $(I)$ increases when distance $\left(f_{l}, c\right)$ decreases. The case when $c$ appears to the right of $f_{r}$ is analogous. Now consider the case where customers appear between $f_{l}$ and $f_{r}$, since the ratio does not increase if customers appear outside of this range (because both OPT and Algorithm Greedy have to consider the region outside this range).

We first consider the case when all customers have costs less than $d$ in the optimum assignment. In the worst case, the adversary places all the customers very close to the facilities except the first customer $c_{1}$ as illustrated in Figure 1. The total cost of Algorithm Greedy is no more than $2|F| d$. In the optimum assignment all customers $c_{i}$ have $\operatorname{cost} \varepsilon_{i}$ except $c_{1}$. The cost of the first customer $c_{1}$ is $\gamma$, where $\gamma>\frac{d}{2}$ (Lemma 1). Then $\frac{\text { Cost_Algorithm_Greedy }(I)}{\text { Cost_OPT }(I)} \leq \frac{2|F| d}{\frac{d}{2}}=4|F|$.

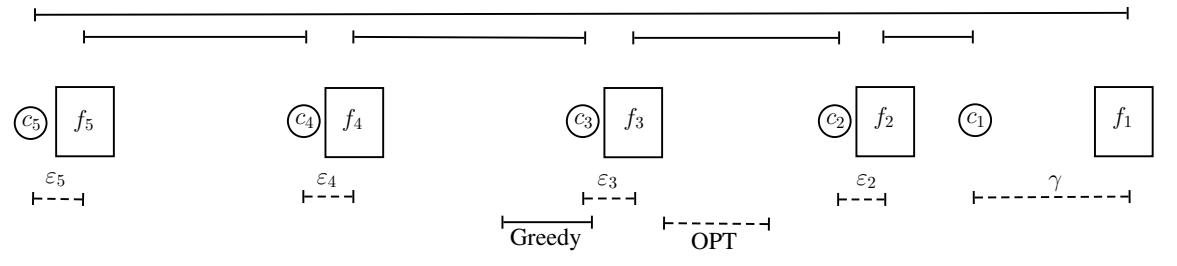

Fig. 1. The configurations of Algorithm Greedy and OPT

In the second case, $k$ customers have costs greater than $d$ in the optimum assignment. Hence, the total cost of the optimum assignment is at least $k d$. We have assumed that the customers at distance less than $d$ are assigned with cost zero by the optimal algorithm and there are $|F|-k$ such customers. In the assignment created by Algorithm Greedy, each of these customers would have cost at most $d$. Note that if any of these customers have cost greater than $d$, then that assignment $A$ can be easily transformed to an equivalent assignment $B$ with total cost equal to that of the original assignment $A$ and so that $|F|-k$ customers have cost no more than $d$. The transformation goes one step at a time, as follows. If a customer $c_{1}$ 
assigned to a facility $f_{1}$ by OPT has cost less than or equal to $d$ and $c_{1}$ is assigned to a facility $f_{2}$ in $A$ and has cost greater than $d$, then we get a new assignment $A^{\prime}$ by assigning $c_{1}$ to $f_{1}$ and $c_{2}$ to $f_{2}$, where $c_{2}$ was the customer assigned to $f_{1}$ in $A$. Similarly, we can swap the next pair to get assignment $A^{\prime \prime}$, and continue this process until we get the equivalent assignment $B$. There are $|F|-k$ customers in $B$ with cost at most $d$ and each of the remaining $k$ customers have a cost at most $(|F|-1) d$. Then $\frac{\text { Cost_Algorithm_Greedy }(I)}{\text { Cost_OPT }(I)} \leq \frac{(|F|-1) d k+(|F|-k) d}{k d}=\frac{(k+1)|F|}{k}-2$.

In the analysis above we assumed unit capacity; now let each facility have capacity $l$, where $l>1$. Suppose that there exists an input sequence of customers $I$ for which the ratio is greater than $4|F|$. We can partition $I$ into $I_{1}, I_{2}, \cdots, I_{l}$ in such a way that the following conditions hold:

$-I_{i} \cap I_{j}=\emptyset$ for $1 \leq i, j \leq l$ and $i \neq j$.

$-I_{1} \cup I_{2} \cup \cdots \cup I_{l}=I$.

- Exactly one customer from $I_{i}$ is assigned to a facility $f_{j}$ for $1 \leq i \leq l$ and $1 \leq j \leq|F|$.

Then there exists a set $I_{\max } \in\left\{I_{1}, I_{2}, \cdots, I_{l}\right\}$ such that the ratio of the corresponding cost of Algorithm Greedy to the cost of OPT is greater than $4|F|$. If we take a set of facilities with unit capacities and place the customers of $I_{\max }$ in the same order as they appear in $I$, the ratio would be greater than $4|F|$ which is a contradiction to the bound of unit capacity.

Note that this algorithm does not generalize to non-equidistant facilities. In particular, if the distances between adjacent facilities increase exponentially, this algorithm can be forced to pay a factor of $O\left(2^{|F|}\right)$ more than OPT.

\subsection{Algorithm $\sigma$-Randomized-Greedy}

In this section we introduce randomness to the greedy method of the previous section and show that better competitive ratios can be obtained.With deterministic online algorithms, the adversary knows the full strategy and can select the worst input sequence. This is not possible if ALG is a randomized algorithm. An oblivious adversary must choose a finite input sequence $I$ in advance. ALG is $c$-competitive against an oblivious adversary if for every such $I$, $E[$ Cost_ALG $(I)] \leq c$.Cost_OPT $(I)+\alpha$ where $\alpha$ is a constant independent of $I$, and $E[$.$] is the mathematical expectation operator taken with respect to the ran-$ dom choices made by ALG. Since the offline player does not know the outcomes of the random choices made by the online player, Cost_OPT $(I)$ is not a random variable and there is no need to take its expectation.

We introduce randomness in Algorithm Greedy, described in the previous section, and call the new method Algorithm $\sigma$-Randomized-Greedy. Let $f_{x}$ be the facility which is nearest to customer $c_{y}$ and let $\sigma$ be a real number. Then $\sigma$ Randomized-Greedy checks whether the distance between $c_{y}$ and $f_{x}$ is less than $\sigma$ and if so then $c_{y}$ is assigned to $f_{x}$. Otherwise, $\sigma$-Randomized-Greedy tosses a fair coin before assigning a customer to a facility, choosing the nearest free facility to the right (left) if the coin comes heads (tails).

We will next show that Algorithm $\sigma$-Randomized-Greedy performs better than Algorithm Greedy. 


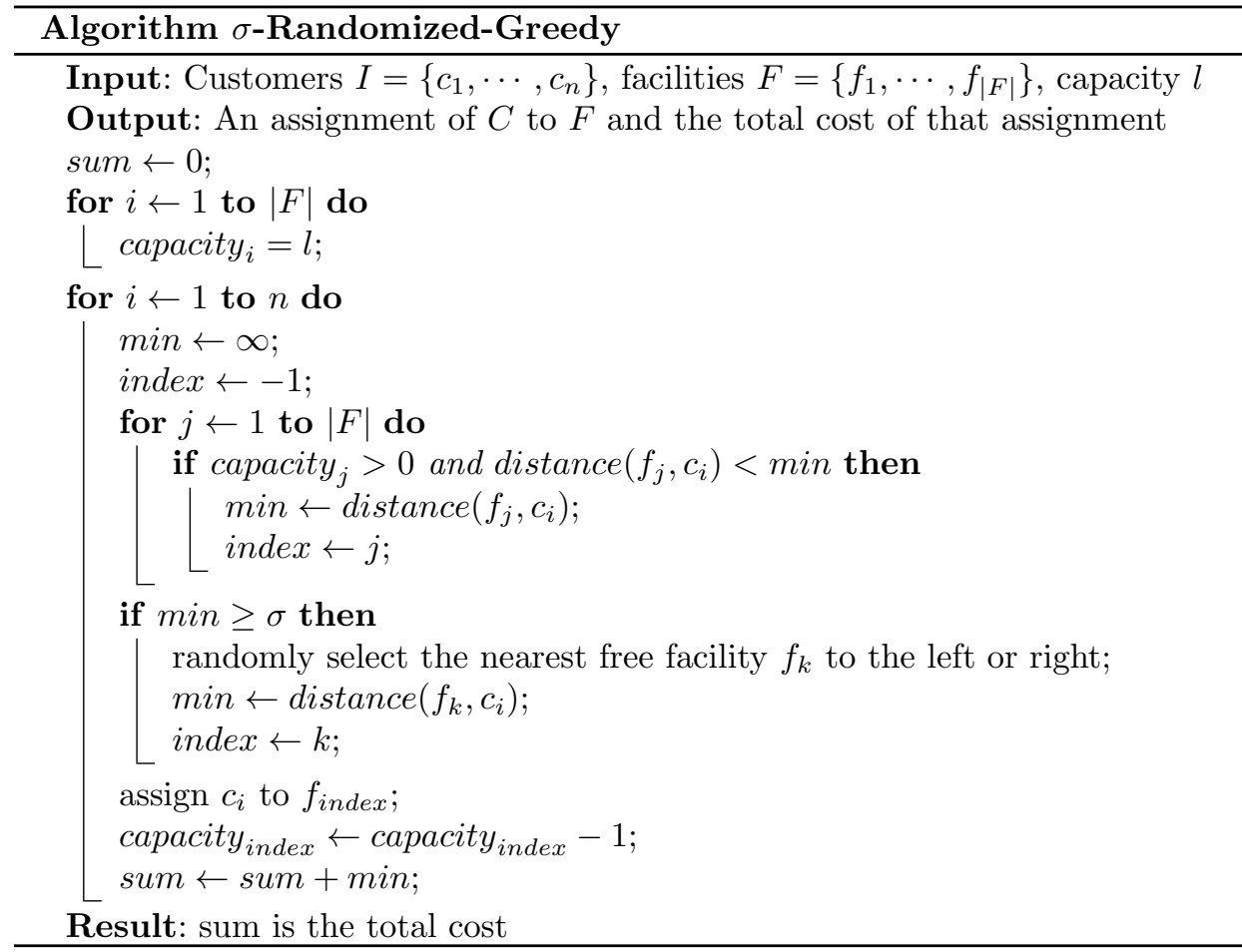

Theorem 2. Let I be a well distributed request sequence for Algorithm Greedy. Let $\gamma$ be the optimal cost for the first customer and $\varepsilon_{i}$ be the optimal cost for $i^{\text {th }}$ customer where $i>1$. If $\sigma>\varepsilon_{i}$ for all $i$ and $\sigma \leq \gamma$ then Algorithm $\sigma$-RandomizedGreedy is $\frac{9}{2}$-competitive for $I$.

Proof. Let $F=\left\{f_{1}, f_{2}, \cdots, f_{|F|}\right\}$ be a set of facilities, such that the distance between every pair of adjacent facilities is $d$, where $d$ is a constant. Recall that if an input $I$ of customers has the property that all assignments cost less than $d$ in the optimum solution, then $I$ is well distributed. The first customer $c_{1}$ is placed closer to $f_{2}$ (Figure 1) in order to fool Algorithm Greedy. Algorithm Greedy assigns $c_{1}$ to $f_{2}$. Except the first customer $c_{1}$, the adversary places every customer $c_{k}$ very close to facility $f_{k}$. Since Algorithm Greedy has already assigned $c_{1}$ to $f_{2}$, it can not assign $c_{2}$ to the same facility. Similarly, for every customer $c_{k}$, Algorithm Greedy assigns it to $f_{k+1}$ although it is very close to $f_{k}$. Algorithm $\sigma$-Randomized-Greedy overcomes this situation by using randomness. Consider the first customer $c_{1}$ who is close to $f_{2}$. Algorithm $\sigma$-Randomized-Greedy chooses either $f_{1}$ or $f_{2}$ with equal probability $\frac{1}{2}$. Similarly for every customer $c_{k}$, Algorithm $\sigma$-Randomized-Greedy chooses either $f_{k+1}$ or $f_{k}$ with equal probability $\frac{1}{2}$. Then

$$
\begin{aligned}
E[\text { Cost_ } \sigma \text {-Randomized-Greedy }(I)]= & \frac{d}{4}+\sum_{i=1}^{|F|-2}\left\{\frac{1}{2^{i+1}}\left(2 i d-\frac{d}{2}\right)\right\} \\
& +\frac{1}{2^{|F|-1}}\left\{2(|F|-1) d-\frac{d}{2}\right\} \\
& <\frac{d}{4}+d \sum_{i=1}^{|F|-2} \frac{i}{2^{i}} \\
& <\frac{d}{4}+2 d \\
= & \frac{9 d}{4}
\end{aligned}
$$


Since the optimum cost is at least $d / 2$, Algorithm $\sigma$-Randomized-Greedy is $\frac{9}{2}$-competitive for $I$.

This shows that Algorithm $\sigma$-Randomized-Greedy can obtain better (expected) competitive ratios than Algorithm Greedy, for appropriate values of $\sigma$. In the theorem above the value of $\sigma$ is very small compared to $d$. If a customer $c_{i}$ is placed beside a facility $f_{j}$ such that the distance between $c_{i}$ and $f_{j}$ is less than $\sigma$, then it is assumed that there is no harm to assign $c_{i}$ to $f_{j}$.

\subsection{Competitive Analysis of Algorithm Optimal-Fill}

In Section 3.1, we showed that Algorithm Greedy can be easily fooled by placing all the customers very close to the facilities except for the first customer. We next describe Algorithm Optimal-Fill, which is more efficient than Algorithm Greedy. The idea is that when a new customer $c_{i}$ arrives, Algorithm Optimal-Fill finds out facility $f_{j}$ that would be selected by an optimal assignment of all the customers $c_{1}, c_{2}, \cdots, c_{i}$. Algorithm Optimal-Fill then assigns $c_{i}$ to $f_{j}$.

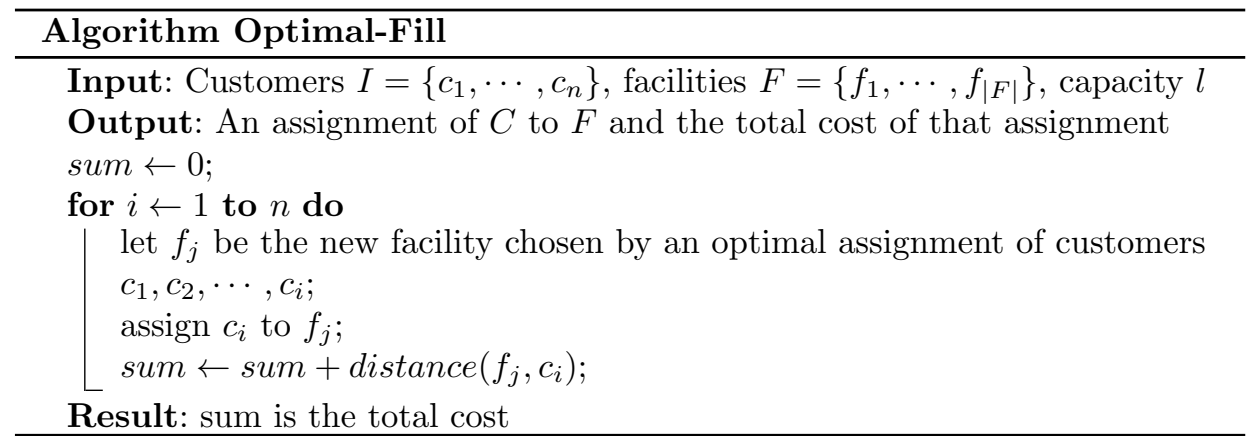

The following theorem shows that Algorithm Optimal-Fill performs better than deterministic greedy method.

Theorem 3. Let $F=\left\{f_{1}, f_{2}, \cdots, f_{|F|}\right\}$ be a set of facilities placed on the line, such that the distance between every pair of adjacent facilities is $d$, where $d$ is a constant. Then $\mathcal{R}$ (Algorithm Optimal-Fill) $\leq|F|$.

Proof. In the worst case, the adversary can place each customer except the first one on top of a facility, so the cost is zero, while Algorithm Optimal-Fill has to pay for each of these customers. The adversary pays only for the first customer and all others are free, because they are placed on top of their facilities. However, Algorithm Optimal-Fill has to pay at least $d$ for each of them. The two algorithms (OPT and Optimal-Fill) are illustrated with an example with 5 facilities and 5 customers in Figure 2.

Then $\frac{\text { Cost_Algorithm_Optimal-Fill }(I)}{\text { Cost_OPT(I) }}=\frac{d+2 d+\cdots+(|F|-1) d+\frac{d}{2}}{\frac{|F| d}{2}}<|F|$

Note that $\mathcal{R}$ (Algorithm Optimal-Fill) is not affected when the distances between adjacent facilities are different. 


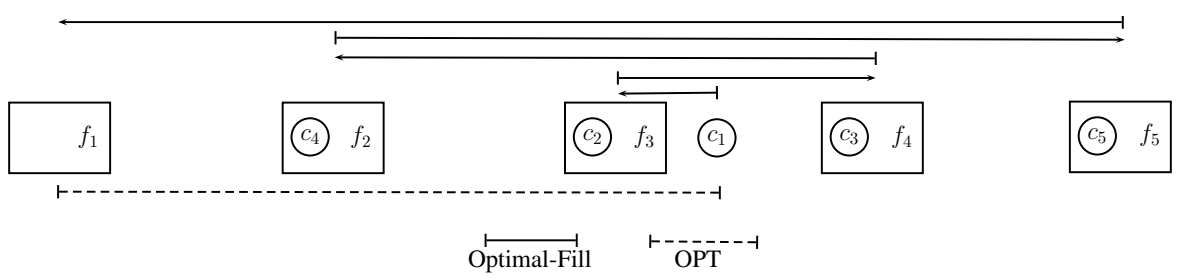

Fig. 2. The adversary places the first customer $c_{1}$ between $f_{3}$ and $f_{4}$. Algorithm OptimalFill assigns $c_{1}$ to $f_{3}$ because it is a little bit closer compared to $f_{4}$. The adversary now places $c_{2}$ exactly on $f_{3}$. Algorithm Optimal-Fill assigns $c_{2}$ to $f_{4}$ because $f_{3}$ and $f_{4}$ are chosen by an optimal assignment for customers $c_{1}$ and $c_{2}$. The adversary then places $c_{3}$ exactly on $f_{4}$. Algorithm Optimal-Fill assigns $c_{3}$ to $f_{2}$ because $f_{2}$ is the new facility chosen by an optimal assignment for customers $c_{1}, c_{2}$ and $c_{3}$.

\section{Facility Assignment on Connected Unweighted Graphs}

We now consider the case where the facilities $F$ are placed on the vertices of a connected unweighted graph $G=(V, E)$ and customers arrive one by one in an online manner at vertices of $G$. We show that Algorithm Greedy has competitive ratio $2|E(G)|$ and Algorithm Optimal-Fill has competitive ratio $|E(G)||F| / r$, where $r$ is the radius of $G$.

\subsection{Competitive Analysis of Algorithm Greedy}

In Section 3.1 we analyzed Algorithm Greedy on a line. The following theorem describes the performance of Algorithm Greedy in the graph setting.

Theorem 4. Let $\mathcal{M}$ be a connected unweighted graph. Then $\mathcal{R}$ (Algorithm Greedy) $\leq$ $2|E(\mathcal{M})|$.

Proof. We assume that the facilities have unit capacity since the analysis is similar for capacity $l$, where $l>1$. Two facilities $f_{i}$ and $f_{j}$ are adjacent if there exists a path $P$ from $f_{i}$ to $f_{j}$ such that no other facilities are situated on $P$. Recall the definition of a well distributed input sequence: an input $I$ is well distributed if there is at least one customer between any two adjacent facilities. We first prove the claim for an input $I$ which is well distributed. Then we show how to transform $I$ to $I^{\prime}$ such that $I^{\prime}$ is well distributed and the competitive ratios of $I$ and $I^{\prime}$ are the same.

We consider two cases; $\mathcal{M}$ is a tree and $\mathcal{M}$ contains at least one cycle. If $\mathcal{M}$ is a tree, we assume that every leaf contains a facility, since $\mathcal{R}$ (Algorithm Greedy) does not increase in the other case. In the worst case Cost_Greedy $(I)$ is less than $2|E(\mathcal{M})|$ and Cost_OPT $(I)$ is equal to one as shown in Figure 3. A square box represents a facility and the input customers are shown by their sequence numbers. In this case competitive ratio is $2|E(\mathcal{M})|$.

If $\mathcal{M}$ contains a cycle, $\mathcal{R}$ (Algorithm Greedy) does not increase. Consider a set of facilities $F$ placed situated on a cycle. In the worst case Cost_Greedy $(I)$ is less than $|E(\mathcal{M})|$ and Cost_OPT $(I)$ is equal to one, as shown in Figure 3. In this case the competitive ratio is $|E(\mathcal{M})|$. 

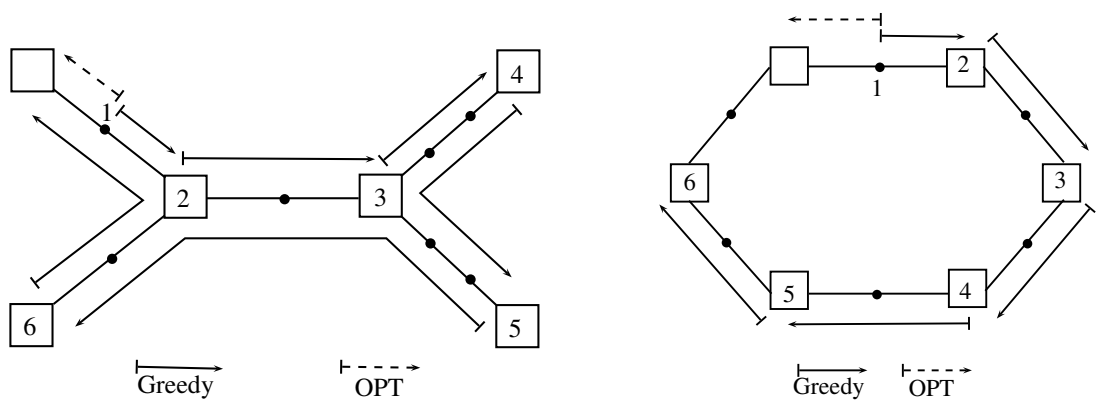

Fig. 3. The configurations of Algorithm Greedy and OPT for a tree and a cycle.

Now suppose the input sequence $I$ is not well distributed. Let $\mathcal{M}^{\prime}$ be the minimum subgraph of $\mathcal{M}$ so that all customers are situated on $\mathcal{M}^{\prime}$. Consider the set of facilities situated on $\mathcal{M}^{\prime}$. In the worst case the customers assigned to those facilities by Algorithm Greedy incur total cost less than $2\left|E\left(\mathcal{M}^{\prime}\right)\right|$ and OPT incurs only unit cost. If OPT incurs cost $x$ to assign a customer to a remaining facility, then Algorithm Greedy incurs at most $x+\left|E\left(\mathcal{M}^{\prime}\right)\right|$ cost to assign a customer to that facility. Hence, Cost_Greedy $(I) \leq$ Cost_OPT $(I)-1+\left|E\left(\mathcal{M}^{\prime}\right)\right|(|E(\mathcal{M})|-$ $\left.\left|E\left(\mathcal{M}^{\prime}\right)\right|\right)+2\left|E\left(\mathcal{M}^{\prime}\right)\right|$. It follows that if $\left|E\left(\mathcal{M}^{\prime}\right)\right|$ is small then Algorithm Greedy will perform similar to OPT. The larger the value of $\left|E\left(\mathcal{M}^{\prime}\right)\right|$ the more well distributed the input $I$ becomes. Hence $\mathcal{R}$ (Algorithm Greedy) $\leq 2|E(\mathcal{M})|$.

Theorem 4 immediately yields the following corollary.

Corollary 1. Let $\mathcal{M}$ be a connected unweighted graph and a set of facilities $F$ is placed on the vertices of $\mathcal{M}$ so that distance between two adjacent facilities is equal. Then $\mathcal{R}($ Algorithm Greedy) $\leq 4|F|$.

\subsection{Competitive Analysis of Algorithm Optimal-Fill}

In Section 3.3 we showed that Algorithm Optimal-Fill was more efficient than Algorithm Greedy, when the metric space was a line. In the case of a connected unweighted graph, it is not straight-forward to determine whether Algorithm Optimal-Fill is better than Algorithm Greedy. The answer depends on the number of edges, facilities and the radius of the graph. The following theorem describes the performance of Algorithm Optimal-Fill.

Theorem 5. Let $\mathcal{M}$ be a connected unweighted graph and a set of facilities $F$ is placed on the vertices of $\mathcal{M}$. Then $\mathcal{R}($ Algorithm Optimal-Fill $) \leq \frac{|E(\mathcal{M})||F|}{r}$.

Proof. The proof is similar to the analysis of Theorem 4. It is sufficient to consider the case when $\mathcal{M}$ is a tree and $I$ is well distributed. Let $x$ be a vertex in the center of $\mathcal{M}$ which is not a facility. If no such vertex exists, the first customer $c_{1}$ is placed on a vertex which is not a facility and the distance from the center of $\mathcal{M}$ is minimum. Otherwise, $c_{1}$ is placed on $x$. In the worst case, Algorithm Optimal-Fill pays a cost equal to the distance between two facilities for each customer, except the first one (see Figure 4). The adversary pays a cost which is no more than radius only for the first customer. Algorithm Optimal-Fill traverses an edge no more than $|F|$ times. Hence, $\mathcal{R}$ (Algorithm Optimal-Fill) is at most $\frac{|E(\mathcal{M})||F|}{r}$. 


\section{Facility Assignment with a Finite Service Time}

Until now we have assumed that if a customer $c_{i}$ is assigned to a facility $f_{j}$, then $c_{i}$ remains there forever. In other words, the service time of an assignment is infinite. Hence a facility with capacity $l$ can provide service to at most $l$ customers. If there are $|F|$ facilities, the total number of customers is limited to $|F| l$. In this section we study the facility assignment problem with a finite service time $t$. We assume a unit time interval between arrivals of customers. When $t=1$, the service time is unit. Let $c_{w}$ be assigned to $f_{x}$. and let us consider the case where all facilities have unit capacities $(l=1)$. If $c_{y}$ is next to $c_{w}$ then we can also assign $c_{y}$ to $f_{x}$ although $c_{w}$ was assigned to $f_{x}$. For unit service time, both Algorithm Greedy and Algorithm Optimal-Fill provide the optimal solution. When the service time is two $(t=2)$, we can not assign $c_{y}$ to $f_{x}$. However, if $c_{z}$ arrives just after $c_{y}$, then we can assign $c_{z}$ to $f_{x}$.

Theorem 6. Let $t$ be the time needed to provide service to a assigned customer. No deterministic algorithm $A L G$ is competitive for $t=2$.

Proof. Let $I=\left(c_{1}, c_{2}, \cdots, c_{n}\right)$ be the input sequence. The adversary places the first customer $c_{1}$ between any two adjacent facilities $f_{i}$ and $f_{i+1}$. Suppose ALG has assigned $c_{1}$ to $f_{i}$. The adversary now places $c_{2}, c_{3}, \cdots, c_{n}$ exactly on the facilities assigned for $c_{1}, c_{2}, \cdots, c_{n-1}$. The adversary runs the optimal algorithm. It assigns $c_{1}$ to $f_{i+1}$, which incurs cost less than $d$, the distance between $f_{i}$ and $f_{i+1}$. The adversary does not pay any cost for the later assignments, because each customer is placed exactly on a facility. However, ALG pays at least $d$ for each assignment except the first one.

\section{Conclusion}

We considered the online facility assignment problem and analyzed several algorithms: Algorithm Greedy, Algorithm $\sigma$-Randomized-Greedy and Algorithm Optimal-Fill. We analyzed the performance of these algorithms in two metric spaces: the 1-dimensional line and a simple, connected, unweighted graph. On the line, we made another strong assumption: that the distance between any two adjacent facilities is the same. The algorithms we describe do not generalize to the case when these distances are arbitrary. In Theorem 2, we further assumed that the input sequence of customers is also well distributed. It would be interesting to find out what happens one or both of these assumptions are dropped. In the graph setting we do not make any assumptions about how the facilities are distributed among the vertices, or about how customers are distributed among the vertices. However, our results in this setting are weaker, in the sense that they depend on 
parameters such as the number of edges in the graph and its radius. A natural question to ask is whether stronger results exist in the graph setting, as well as in other metric spaces.

\section{References}

1. Akagi, T., Nakano, S.i.: On r-gatherings on the line. In: Wang, J., Yap, C. (eds.) Frontiers in Algorithmics, Lecture Notes in Computer Science, vol. 9130, pp. 25-32. Springer International Publishing (2015)

2. Antoniadis, A., Barcelo, N., Nugent, M., Pruhs, K., Scquizzato, M.: Approximation and Online Algorithms: 12th International Workshop, WAOA 2014, Wrocław, Poland, September 11-12, 2014, Revised Selected Papers, chap. A $o(n)$-Competitive Deterministic Algorithm for Online Matching on a Line, pp. 11-22. Springer International Publishing, Cham (2015)

3. Armon, A.: On min-max r-gatherings. Theoretical Computer Science 412(7), 573-582 (2011)

4. Bansal, N., Buchbinder, N., Gupta, A., Naor, J.S.: An $O\left(\log ^{2} k\right)$-competitive algorithm for metric bipartite matching. Algorithmica 68(2), 390-403 (2012)

5. Bartal, Y., Koutsoupias, E.: On the competitive ratio of the work function algorithm for the k-server problem. Theoretical Computer Science 324(23), 337 - 345 (2004)

6. Bein, W.W., Chrobak, M., Larmore, L.L.: The 3-server problem in the plane. Theoretical Computer Science 289(1), 335 - 354 (2002)

7. Chrobak, M., Karloff, H., Payne, T., Vishwanathan, S.: New results on server problems. In: SIAM Journal on Discrete Mathematics. pp. 291-300 (1990)

8. Chrobak, M., Larmore, L.L.: An optimal on-line algorithm for k-servers on trees. SIAM Journal on Computing 20(1), 144-148 (Feb 1991)

9. Drezner, Z.: Facility Location: A Survey of Applications and Methods. springer (1995)

10. Drezner, Z., Hamacher, H.W.: Facility Location: Applications and Theory. springer (2004)

11. Kalyanasundaram, B., Pruhs, K.: Online weighted matching. Journal of Algorithms 14(3), $478-488(1993)$

12. Kao, M.Y., Reif, J.H., Tate, S.R.: Searching in an unknown environment: An optimal randomized algorithm for the cow-path problem. Information and Computation 131(1), 63 - 79 (1996)

13. Khuller, S., Mitchell, S.G., Vazirani, V.V.: On-line algorithms for weighted bipartite matching and stable marriages. Theoretical Computer Science 127(2), 255 - 267 (1994)

14. Kleinberg, J.M.: A lower bound for two-server balancing algorithms. Information Processing Letters 52(1), 39 - 43 (1994)

15. Koutsoupias, E., Papadimitriou, C.: The 2-evader problem. In: Information Processing Letters. pp. 473-482 (1996)

16. Manasse, M.S., McGeoch, L.A., Sleator, D.D.: Competitive algorithms for server problems. Journal of Algorithms 11(2), 208-230 (May 1990)

17. Schrijver, A.: Combinatorial Optimization: Polyhedra and Efficiency, Algorithms and Combinatorics, vol. 24. Springer, Berlin (2003)

18. Sleator, D.D., Tarjan, R.E.: Amortized efficiency of list update and paging rules. Communications of the ACM 28(2), 202-208 (Feb 1985) 